\title{
A DFT study of hydroxyapatite (001) surface
}

\author{
Albert F. B. Bittencourt ${ }^{1 *}$ (albertbittenc@gmail.com), Rogério Custodio ${ }^{2}$, \\ Gustavo P. Valença ${ }^{3}$ \\ ${ }^{1}$ Faculty of Chemical Engineering, State University of Campinas, 13083-852, Campinas, SP, Brazil. \\ ${ }^{2}$ Institute of Chemistry, State University of Campinas, 13083-861, Campinas, SP, Brazil. \\ ${ }^{3}$ Faculty of Chemical Engineering, State University of Campinas, 13083-852, Campinas, SP, Brazil. \\ Keywords: hydroxyapatite, surface energy, density functional theory
}

\section{Introduction}

Hydroxyapatite $\left(\mathrm{Ca}_{10}\left(\mathrm{PO}_{4}\right)_{6}(\mathrm{OH})_{2}-\mathrm{HAP}\right)$ is a mineral commonly employed in bone regeneration ${ }^{(1)}$. Due to the presence of both acid and basic sites in a single crystal lattice, this solid also is used as a catalyst ${ }^{(2)}$. Several experimental studies have investigated the acid-base properties of HAP. However, there is still no consensus on the involvement of these sites and adsorbed molecules at a microscopic level ${ }^{(3)}$. In this sense, this work investigates the distribution of the acid-base sites of HAP (001) surface using DFTcalculations.

\section{Methodology}

The total energies of the bulk unit cell and (001) surface slab model of hydroxyapatite were estimated using the open-source code QUANTUM ESPRESSO ${ }^{(4)}$. The PAW method ${ }^{(5)}$ was employed with the GGA PBE functional ${ }^{(6)}$ for an energy cutoff of 48 Ry and a Monkhorst-Pack ${ }^{(7)}$ k-point mesh of $4 \times 4 \times 4$ for the bulk unit cell and $4 \times 4 \times 1$ for the slab model. The convergence criteria for the total energy, force per atom, and pressure was set to $0.1 \mathrm{mRy}, 1 \mathrm{mRy} \cdot \mathrm{bohr}^{-1}, 0.5 \mathrm{kbar}$, respectively. The (001) surface was modeled as a double layer slab separated by a vacuum width of $20 \AA$. The VESTA software ${ }^{(8)}$ was applied to build and visualize the structural model and the surface potential map.

\section{Results}

After a geometry optimization of both lattice parameters and atomic coordinates, the cell dimensions were computed to be $\mathrm{a}=\mathrm{b}=9.562 \AA$ and $\mathrm{c}=6.896 \AA$.

The surface energy $\left(E_{\text {surf }}\right)$ was calculated as

$$
E_{\text {surf }}=\frac{E_{\text {slab }}-n E_{\text {bulk }}}{2 A}
$$

in which $E_{\text {slab }}$ is the energy of the slab model, $n$ the number of bulk unit cells, $E_{\text {bulk }}$ the energy of an individual bulk unit cell and $A$ the surface area per unit cell. A value of $0.714 \mathrm{~J} \cdot \mathrm{m}^{-2}$ was encountered for the $(001)$ relaxed surface.

The (001) surface electrostatic potential was also computed from -0.15 to 0.15 a.u. for a constant electron density of $5 \times 10^{-4}$ a.u. (see Figure 1 ).

\section{Discussion}

The lattice parameters here calculated are in good agreement with the experimental values of $\mathrm{a}=\mathrm{b}=9.417 \AA$ and $\mathrm{c}=6.875 \AA^{(9)}$.

Even though no experimental measurements of surface energy are available in the literature for comparison, previous simulation studies encountered $0.871 \mathrm{~J} \cdot \mathrm{m}^{-2}$ (VASP code) ${ }^{(10)}, 1.043 \mathrm{~J} \cdot \mathrm{m}^{-2}\left(\right.$ CRYSTAL03 code) ${ }^{(11)}$, and $1.147 \mathrm{~J} \cdot \mathrm{m}^{-2}($ CRYSTAL14 code) ${ }^{(12)}$ for HAP (001) surface. 
In the electrostatic potential map (Figure 1) it is possible to distinguish between positive and negative potential zones which correspond to the calcium ions and the oxygen atoms of the phosphate groups, respectively. This result relates to experimental evidence that acid and basic sites are distributed over HAP surface, then suggesting that the calcium ions and the oxygen of phosphate groups may play an important role during the adsorption process of molecules.

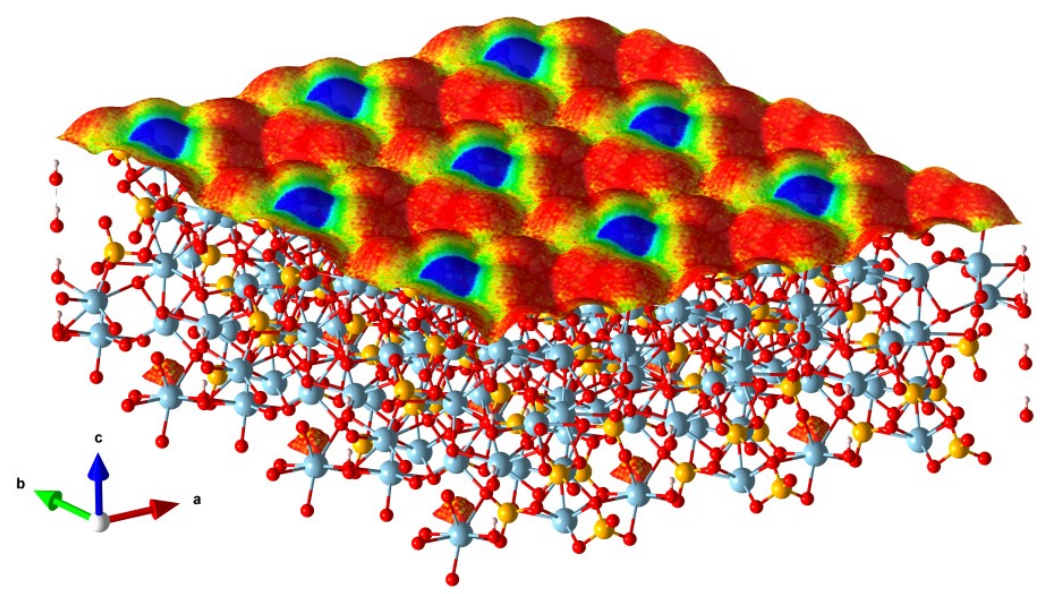

Figure 1. Electrostatic potential mapped onto the (001) surface of HAP. Positive and negative potential zones are presented in blue and red, respectively. Six unit cells were considered for this map.

\section{Conclusions}

This work presented a computational simulation of HAP (001) surface by using a double layer slab model. The surface energy showed a lower value when compared to other studies which is probably related to different methods and codes employed. An important observation from the surface potential map is that positive and negative zones are related to the distribution of calcium and phosphates groups on the surface. More detailed studies are necessary to clarify the interaction between these sites and other molecules relevant to catalytic processes.

\section{Acknowledgments}

The authors thank the Center for Computational Engineering \& Sciences (CCES) at the University of Campinas (UNICAMP) and the Fundação de Amparo à Pesquisa do Estado de São Paulo (FAPESP) for the computational support (process number 2013/08293-7).

\section{References}

(1) Liu, H. et al. Biomaterials, 2013, 34, 4404.

(2) Tsuchida, T. et al. J. Catal., 2008, 259, 183.

(3) Diallo-Garcia, S. et al. J. Phys. Chem. C, 2014, 118, 12744.

(4) Giannozzi, P. et al. J. Phys.: Condens. Matter, 2017, 29, 465901.

(5) Blochl, P. E. Phys. Rev. B, 1994, 50, 17953.

(6) Perdew, J. P., Burke, K., Ernzerhof, M. Phys. Rev. Lett., 1996, 77, 3865.

(7) Monkhorst, J., Pack, J. D. Phys. Rev. B, 1976, 13, 5188.

(8) Momma, K., Izumi, F. J. Appl. Crystallogr., 2011, 44, 1272.

(9) Hughes, J. M., Cameron, M., Crowley, K. D. Am. Mineral., 1989, 74, 870.

(10) Rulis, P. et al. Phys. Rev. B, 2007, 76, 245410.

(11) Corno, M. et al. Eur. J. Mineral., 2007, 19, 757.

(12) Ulian, G., Moro, D., Valdrè, G. J. Appl. Crystallogr., 2016, 49, 1893. 